\title{
A TWO-PART DEFENSE OF INTUITIONISTIC MATHEMATICS
}

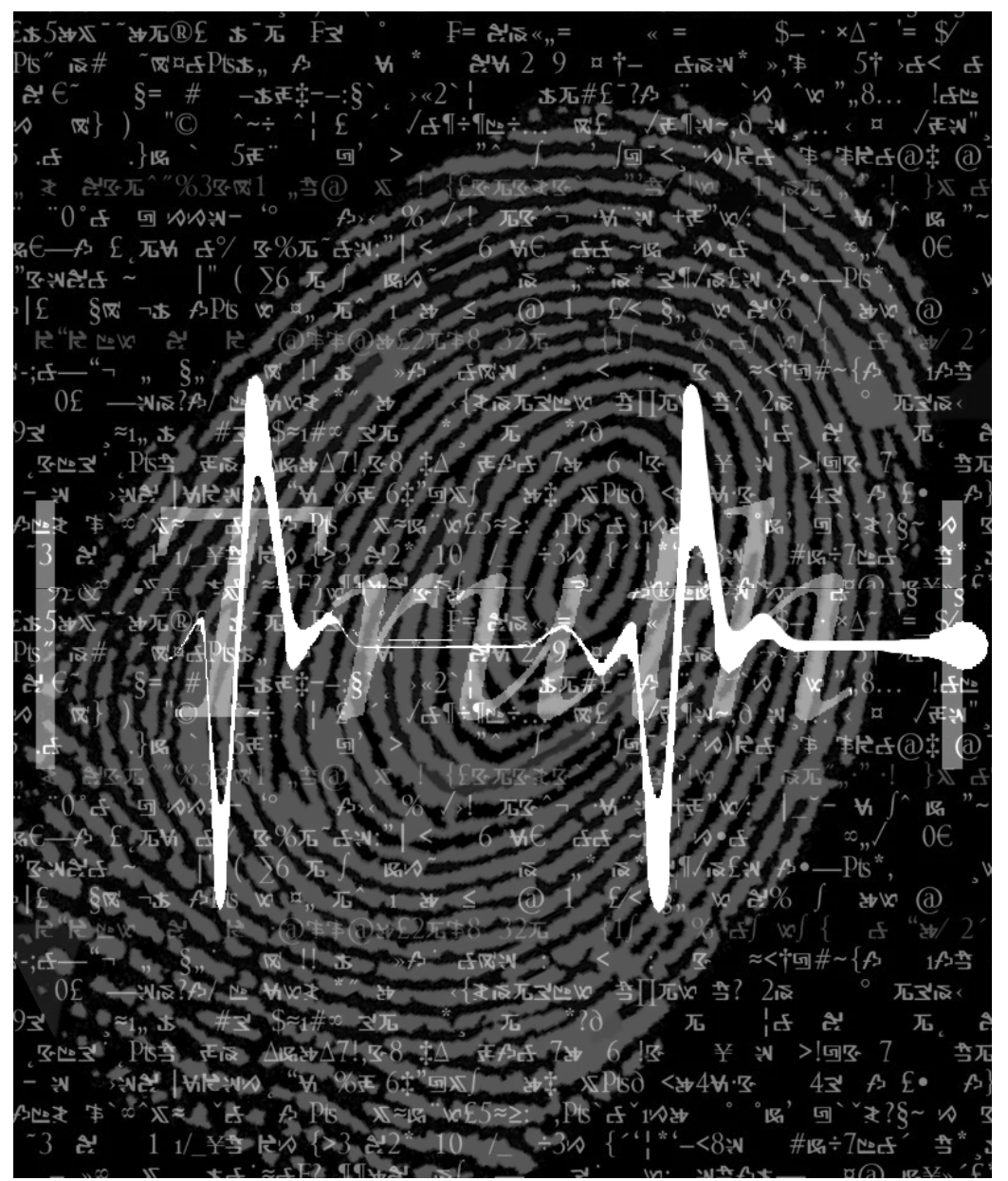

\section{ABSTRACT}

The classical interpretation of mathematical statements can be seen as comprising two separate but related aspects: a domain and a truth-schema. L. E. J. Brouwer's intuitionistic project lays the groundwork for an alternative conception of the objects in this domain, as well as an accompanying intuitionistic truth-schema. Drawing on the work of Arend Heyting and Michael Dummett, I present two objections to classical mathematical semantics, with the aim of creating an opening for an alternative interpretation. With this accomplished, I then make the case for intuitionism as a suitable candidate to fill this void.

SAMUEL R. ELLIOTT 
Any justification for adopting one logic rather than another as the logic for mathematics must turn on questions of meaning.

-Michael Dummett

"The Philosophical Basis of Intuitionistic Logic"

\section{INTRODUCTION}

As Arend Heyting and Michael Dummett present it, mathematics, when interpreted classically, is concerned with issues of fundamental (which is to say, metaphysical) mathematical truths. The assumption which underlies this interpretation is that there exists a "realm of mathematical reality, existing objectively," "some world of mathematical things existing independently of our knowledge," which mathematics is concerned with studying. ${ }^{2}$

To put this another way, the assumption which underpins the classical interpretation of mathematical statements is that the domain of natural numbers $\mathbf{N}$ is populated with objectively existing, mindindependent abstract objects; the referring-terms of our mathematical language (i.e., the natural number names) refer to these objects, which determinately satisfy or fail to satisfy certain properties. In short, classical mathematicians are realists about numbers. This assumption is metaphysical - it concerns the status of being of the members of $\mathbf{N}$, the quantificational domain of our mathematical language.

In introducing his defense of L. E. J. Brouwer's intuitionistic project, Heyting insists that such metaphysical questions should not be made to bear on mathematical issues. ${ }^{3}$ Intuitionism takes it as an uncontroversial fact that we do practice mathematics and that we do so in certain ways. The project of intuitionism, as Heyting presents it, is to study just this process of doing mental mathematics; that is, intuitionistic mathematics is the study of mental mathematical construction as such, without assuming anything about the fundamental metaphysical nature of mathematical objects. ${ }^{4}$

Michael Dummett, Elements of Intuitionism, 2nd ed. (Oxford: Clarendon Press, 2000), 5.

Arend Heyting, Intuitionism: An Introduction (Amsterdam: North-Holland, 1956), 3.

3 "Mathematics ought not to depend upon such [metaphysical] notions as these." See Heyting, Intuitionism, 3.

4 "Brouwer's program ... consisted in the investigation of mental mathematical construction as such, without reference to questions regarding the nature of the constructed objects, such as whether these objects exist independently of our knowledge of them." See Heyting, Intuitionism, 1.
Dummett seconds this articulation of intuitionistic mathematics in the introductory remarks of his Elements of Intuitionism. ${ }^{5}$

In other words, the intuitionistic interpretation of our mathematical language begins from the rejection of the metaphysical assumption underlying classical mathematics. On an intuitionistic interpretation, the domain of our mathematical language is still the set of natural numbers $\mathbf{N}$, but the members of $\mathbf{N}$ should be viewed as "mental mathematical constructions." For our purposes, we may understand "mental mathematical constructions" as being preanalytical ideas (perhaps of some requisite clarity or distinctness): that is, mind-dependent entities. Dummett, again, seconds this analysis: "To an intuitionist ... mathematical objects themselves are mental constructions ... They exist only in virtue of our mathematical activity, which consist in mental operations." 6

I shall here present intuitionistic mathematics as differing primarily from classical mathematics over the interpretation of the meaning of mathematical statements. This issue of meaning can be decomposed into two related issues: what is the domain of our mathematical language and what is its truth-schema? I shall begin by briefly outlining the classical interpretation of mathematical statements and how this interpretation addresses these two questions. Drawing on Heyting, I will then make an Ockhamian case for preferring an intuitionistic domain to a classical domain on the grounds of ontological parsimony. I will then argue, following Dummett, that we ought also to replace the classical truth-schema-rooted in a notion of fundamental (metaphysical) truth-with an intuitionistic schema rooted in the notion of proof. I will conclude by considering some objections and possible replies.

\section{CLASSICAL SEMANTICS}

Following Dummett's lead I will take a "mathematical statement" to be any statement which takes as its referring-terms, the natural numbers N.7 For simplicity, we may confine our discussion to a toy mathematical language $\boldsymbol{L}$, whose domain is the set of natural numbers $\mathbf{N}$, containing only a few classes of sentences:

5 "To an intuitionist ... mathematical objects themselves are mental constructions ... They exist only in virtue of our mathematical activity, which consist in mental operations, and have only those properties which they can be recognized by us as having." See Dummett, Elements of Intuitionism, 5.

6 Dummett, Elements of Intuitionism, 5.

7 Michael Dummett, "The Philosophical Basis of Intuitionistic Logic," in Truth and Other Enigmas (Cambridge: Harvard University Press, 1978), 215 
Predicate-subject sentences of the form " $\alpha$ is $\pi$," where " $\alpha$ " is a referring-term, and " $\pi$ " is a (unary) predicate:

E.g., "7 is a prime number;"

Negations of the form "not $\varphi$," where " $\varphi$ " is a sentence:

E.g., "4 is not a prime number;"

Existential sentences of the form "some $\mathrm{x}$ is $\pi$," where " $\mathrm{x}$ " is a variable and " $\pi$ " is a predicate:

E.g., the Ordinary Perfect Number Conjecture (OPN):

"There is an odd perfect number (equal to the sum of its positive non-equal divisors);"

Universal statements of the form "every $\mathrm{x}$ is $\pi$," where " $\mathrm{x}$ " is a variable and " $\pi$ " is a predicate:

E.g., Goldbach's Conjecture: "every even integer greater than 2 can be expressed as the sum of two primes."

Formally, the sentences of $\boldsymbol{L}$ can be defined recursively as follows:

$$
\varphi::=\pi(\alpha)|\neg \varphi| \exists x \varphi \mid \operatorname{Vx} \varphi
$$

As we have said, the assumption which underpins the classical interpretation is that the domain of natural numbers $\mathbf{N}$ is populated with objectively existing, mind-independent abstract objects, to which the referring-terms of $\boldsymbol{L}$ refer, and which determinately satisfy or fail to satisfy certain properties. On the basis of this assumption, we can formulate an inductive definition of truth (in $\boldsymbol{L}$ ), in the form of a Tarskian truth-schema, using a metalinguistic T-predicate (ranging over the sentences of $\boldsymbol{L}$ ) as follows: ${ }^{9}$

" $\alpha$ is $\pi$ " is $\mathbf{T}$ iff $\alpha$ (the member of $\mathbf{N}$ to which " $\alpha$ " refers) is $\pi$;

"Not $\varphi$ " is $\mathbf{T}$ iff $\varphi$ is not $\mathrm{T}$;

"Some $\mathrm{x}$ is $\pi$ " is $\mathbf{T}$ iff some member of $\mathbf{N}$ is $\pi$;

"Every $\mathrm{x}$ is $\pi$ " is $\mathbf{T}$ iff every member of $\mathbf{N}$ is $\pi$.

This schema can be synthesized with our previous assumption about the metaphysical nature of the members of the domain $\mathbf{N}$ to give the following generalization of the classical interpretation of mathematical sentences:

" $\varphi$ " is $\mathbf{T}$ iff $\varphi$ is true (in some metaphysically-committed sense);

8 Christian Goldbach, Letter to Leonhard Euler, June 7, 1742.

9 Alfred Tarski, "The Semantic Conception of Truth: And the Foundations of Semantics," Philosophy and Phenomenological Research 4, no. 3 (1944): 341-76, $10.2307 / 2102968$
Following Gottlob Frege's claim that the reference of a sentence is its truth-value, we can give the following restatement of this generalization (which, for our purposes, we may take as equivalent): ${ }^{10}$

" $\varphi$ " means that $\varphi$ is true (in some metaphysically-committed sense).

\section{HEYTING: AN OCKHAMIAN OBJECTION}

If "to exist" does not mean "to be constructed," it must have some metaphysical meaning. It cannot be the task of mathematics to investigate this meaning or to decide whether it is tenable or not. -Arend Heyting, Intuitionism: An Introduction

The first concern I would like to raise regarding the viability of this classical interpretation concerns the initial assumption that the domain of our mathematical language should be populated by mindindependent, abstract objects, existing in a transcendent realm of mathematical reality. Rather than attempt to refute this assumption directly, I would simply argue, following Heyting, that it is neither desirable nor necessary to found mathematics on such metaphysical assumptions. ${ }^{11}$

By virtue of being realist about numbers, the classical mathematician's interpretation of $\mathbf{N}$ requires a supporting ontology containing numbers as a sui generis class of mind-independent entity. Following Ockham's razor-the principle that "entities should not be multiplied beyond necessity" - we should hold an interpretation of $\mathbf{N}$ which did not require such ontological commitments to be theoretically preferable (on this count, at least). ${ }^{12}$

Moreover, this is precisely what the intuitionistic mathematician offers; with an intuitionistic interpretation, the domain of our mathematical language is still the set of natural numbers $\mathbf{N}$, but the members of $\mathbf{N}$ should be viewed as "mental mathematical constructions." Returning to our toy mathematical language $\boldsymbol{L}$, the intuitionist holds that, as the members of the domain $\mathbf{N}$, it is these mental mathematical constructions to which referring terms like " 7 " refer, which satisfy predicates such as "is prime," and over which the existential and universal quantifiers " $\exists$ " and " $\mathrm{V}$ " quantify.

Of course, the ontology required to support this interpretation must include "mental mathematical constructions," which may seem

10 Gottlob Frege, "Sense and Reference," The Philosophical Review 57, no. 3 (1948): 209-30, 10.2307/2181485.

11 Heyting, Intuitionism, 3.

12 Alan Baker, "Simplicity," in The Stanford Encyclopedia of Philosophy (Winter 2016 Edition), ed. Edward N. Zalta, https://plato.stanford.edu/archives/ win2016/entries/simplicity/ 
dubious, especially in the absence of a precise definition. However, as we have presented it, "mental mathematical constructions" are, essentially, ideas (or a subclass thereof), and I would venture that any serious classical mathematician would acquiesce to an ontology containing such entities as ideas (in their mental ontology, if not in their mathematical ontology).

This argument is hardly conclusive, nor does it leave us with a clear indication of how we are to understand the sentences in our mathematical language. How, for instance, are we to understand the attribution of the property "being prime" to the number 7, qua mental construction? However, it does lend some theoretical weight to the choice of an intuitionistic domain over a classical domain. As Heyting puts it, it is a primitive psychological fact that we do practice mathematics: to found this on a basis of metaphysical assumption is both unnecessary and undesirable, for our conclusions are only as certain as the assumptions which ground them. ${ }^{13}$

\section{DUMMETT: A WITTGENSTEINIAN OBJECTION}

A stronger repudiation of the classical interpretation of our mathematical language can be found in Dummett's "Philosophical Basis," directly attacking the viability of the classical truth-schema. Dummett's argument draws heavily on Ludwig Wittgenstein's Philosophical Investigations, particularly the doctrine that "the meaning of a word is its use in the language" 14 and "The meaning of a mathematical statement determines and is exhaustively determined by its use." 15 If use determines meaning, it follows (by something similar to the causal adequacy principle) that the meaning of a sentence cannot consist in anything which is not present and manifest in its use. The justification for this doctrine is that it does not make sense to talk about a statement as having meaning when divorced from its linguistic context, no more than it makes sense to say of a chess piece that it has particular powers ("it can move any number of squares diagonally but cannot leap over other pieces") when removed from the context of a game of chess. ${ }^{16}$

13 "We have no objection against a mathematician privately admitting any metaphysical theory he likes, but Brouwer's program entails that we study mathematics as something simpler, more immediate than metaphysics." See Heyting, Intuitionism, 2.

14 Ludwig Wittgenstein, Philosophical Investigations, trans. G. E. M. Anscombe (New York: MacMillan, 1953), 43

15 Dummett, "Philosophical Basis," 216.

16 Dummett, "Philosophical Basis," 216.
Recall, from our consideration of the toy language $\boldsymbol{L}$, we inductively derived the following general classical truth-schema for our mathematical language:

" $\varphi$ " is $\mathbf{T}$ iff $\varphi$ is true (in some metaphysically-committed sense). Following Frege's claim that the reference of a sentence is its truthvalue, we then gave the following (roughly) equivalent restatement:

" $\varphi$ " means that $\varphi$ is true (in some metaphysically-committed sense). However, returning to consideration of $\boldsymbol{L}$, we can show that the truthvalues of existential and universal sentences in $\boldsymbol{L}$ are not necessarily decidable under the classical truth-schema. To make this argument, we must be clear in drawing a distinction between the $\mathbf{T}$-predicate we introduced earlier - a predicate ranging over sentences in $\boldsymbol{L}$ — and (what we have called) "truth," in the metaphysically-loaded sense.

Let us assume that we have a decidable predicate $\mathrm{F}$, such that for any given $\mathrm{x} \in \mathbf{N}$ produced, we can determine whether $\mathrm{F}(\mathrm{x})$ or $\neg \mathrm{F}(\mathrm{x})$ actually obtains - that is to say, whether $\mathrm{F}(\mathrm{x})$ or $\neg \mathrm{F}(\mathrm{x})$ is true, and hence, whether " $\mathrm{F}(\mathrm{x})$ " or " $\mathrm{F}(\mathrm{x})$ " is $\mathbf{T}$. Given that we take the universal quantifier "V" as quantifying over an infinite domain $\mathbf{N}$, we cannot verify a posteriori (by cases) that " $\mathrm{VxF}(\mathrm{x})$ " is $\mathbf{T}$, as it is not possible to individually confirm for every $\mathrm{x} \in \mathbf{N}$ that $\mathrm{F}(\mathrm{x})$ is true, nor, by the same token, that " $\mathrm{F}(\mathrm{x})$ " is $\mathbf{T}$. Nor is it necessarily possible to verify a priori that " $\mathrm{VxF}(\mathrm{x})$ " is $\mathbf{T}$ : for instance, we might assume that $\mathrm{F}(\mathrm{x})$ obtains for every $\mathrm{x}$, but does so purely by accident or coincidence, and hence that there exists no finite set of reasons for determining that $\mathrm{F}(\mathrm{x})$ obtains for every $\mathrm{x}$. In such a scenario, " $\mathrm{VxF}(\mathrm{x})$ " would be $\mathbf{T}$, but would not be verifiable a priori. Hence, the $\mathbf{T}$ universal sentences of $\boldsymbol{L}$ cannot necessarily (which is to say, in general) be verified either a posteriori or a priori. ${ }^{17}$

Similarly, falsifying an existential sentence " $\exists \mathrm{xF}(\mathrm{x})$ " is equivalent to verifying its negation " $\neg \exists \mathrm{xF}(\mathrm{x})$," which is in turn equivalent to verifying the universal " $\mathrm{Vx}_{\neg} \mathrm{F}(\mathrm{x})$." By the same logic as above, we cannot verify " $\mathrm{V}_{\mathrm{X}} \mathrm{F}(\mathrm{x})$ " a posteriori nor necessarily a priori, even if it is $\mathbf{T}$, and hence, it follows that we cannot necessarily falsify the not-T existential sentences of $\boldsymbol{L}$.

It follows that, under the classical truth-schema for $\boldsymbol{L}$, we are not in general capable of verifying that a $\mathbf{T}$ universal sentence of $\boldsymbol{L}$ is $\mathbf{T}$, nor that a not- $\mathbf{T}$ existential sentence of $\boldsymbol{L}$ is not- $\mathbf{T}$. This conclusion generalizes outside of $\boldsymbol{L}$, to our full mathematical language.

As such, it is not clear how the actual truth of a mathematical statement could manifest itself in our use of that statement, given that

17 Dummett, Elements of Intuitionism, 3. 
we are not, in general, capable of recognizing that these conditions obtain when they obtain. To put this anecdotally: if the actual truth (or falsity) of a mathematical statement is beyond our knowledge, what difference could its being true or false make to how we use the statement? "This conception violates the principle that use exhaustively determines meaning." 18

The problem is that, if use determines meaning, and the meaning of a sentence cannot consist in anything which is not present and manifest in its use, then it is unclear how the notion of truth can figure in any effective exposition of the meaning of a large class of sentences in our mathematical language. Hence, if we accept the Wittgensteinian doctrine that meaning is use, we should reject the classical mathematical semantics rooted in the notion of truth (at least as it applies to universals and existentials).

Note, our argument here need not give way to general skepticism about mathematical truths. We may concede that, for a particular number, $\alpha$, and a decidable predicate, $\pi$, there is no problem in assuming that a grasp of the truth or falsity of $\pi(\alpha)$ could determine our use of the sentence " $\pi(\alpha)$," and hence, that such predicate-subject statements, interpreted classically, could be meaningful. The problem is that mathematics, as a subject, concerns itself with the investigation and assertion of universal and existential claims about the set of natural numbers $\mathbf{N}$, whose truth is, as we have shown, in general, undecidable.

\section{INTUITIONISTIC SEMANTICS}

This argument, of course, does not constitute a positive argument for adopting an intuitionistic interpretation of our mathematical language, but only gives grounds for rejecting the classical interpretation. Nevertheless, it does give a clear criterion for adequacy in mathematical semantics: the meaning of our mathematical language must be rooted in some decidable property, such that, in general, we are able to know that a mathematical sentence is $\mathbf{T}$ when it is $\mathbf{T}$.

Again, intuitionism offers a solution here. As Dummett puts it, "We must, therefore, replace the notion of truth, as the central notion of the theory of meaning for mathematical statements, by the notion of proof: a grasp of the meaning of a statement consists in a capacity to recognize a proof of it when one is presented to us." ${ }^{19}$

On this basis, we can formulate an alternative inductive definition of truth (in our toy language $\boldsymbol{L}$ ), as follows:

Dummett "Philosophical Basis," 224

19 Dummett, "Philosophical Basis," 225.
" $\alpha$ is $\pi$ " is $\mathbf{T}$ iff it is proven that $\alpha$ is $\pi$;

"Not $\varphi$ " is $\mathbf{T}$ iff it is proven that not- $\varphi$;

"Some $\mathrm{x}$ is $\pi$ " is $\mathbf{T}$ iff it is proven that some member of $\mathbf{N}$ is $\pi$;

"Every $\mathrm{x}$ is $\pi$ " is $\mathbf{T}$ iff it is proven that every member of $\mathbf{N}$ is $\pi$. In general:

" $\varphi$ " is $\mathbf{T}$ iff $\varphi$ is proven;

And hence:

" $\varphi$ " means that $\varphi$ is proven.

To see how this schema avoids the pitfalls which troubled the classical schema, we should reintroduce the conclusion of our previous argument - that the domain of our mathematical language is populated with mental mathematical constructions.

On an intuitionistic interpretation, an existential statement, " $\exists x F(x)$, " means that we have constructed a proof of $\exists x F(x)$. On a Brouwer, Heyting, and Kolmogorov (BHK) interpretation, this is tantamount to saying that we have constructed at least one example where $x \in \mathbf{N}$ and a demonstration (proof) that $\mathrm{F}(\mathrm{x})$ for that $\mathrm{x}$. For example, "There exists an odd perfect number" (OPN) means that we have constructed an example where $\mathrm{x} \in \mathbf{N}$, and a proof that $\mathrm{x}$ is both perfect and odd. Since we have not effected such a construction, it follows that OPN (interpreted intuitionistically) is not-T.

Similarly, a universal statement " $\mathrm{VxF}(\mathrm{x})$ " means that we have constructed a proof of $\operatorname{VxF}(\mathrm{x})$ : that is, a function which maps (or would map) each $\mathrm{x} \in \mathbf{N}$ to a proof of $\mathrm{F}(\mathrm{x})$. For example, "every even integer greater than two can be expressed as the sum of two primes" means that we have a construction which maps (or would map) every constructed $\mathrm{x}$ which is both even and greater than two (this being a subset of $\mathbf{N}$ ) to a proof that x can be expressed as the sum of two primes. Since no such construction has yet been effected, it follows that Goldbach's Conjecture (interpreted intuitionistically) is not-T. ${ }^{20}$

To my mind, this intuitionistic account adequately circumvents the decidability issue which had destabilized the classical interpretation. With clear standards for what constitutes a proof of a universal or existential mathematical statement, it should be generally decidable whether a given statement is proven or unproven. Hence, there is no

20 "A mathematical assertion affirms the fact that a certain mathematical construction has been effected." See Heyting, Intuitionism, 3; "From an intuitionistic standpoint, therefore, an understanding of a mathematical statement consists in a capacity to recognize a proof of it when presented with one; and the truth of such a statement consist only in the existence of such a proof." See Dummett, Elements of Intuitionism, 4. 
obvious issue with supposing that such a notion of proof should figure in our use of such mathematical statements, or that such a notion should ground the meaning of our mathematical language.

\section{OBJECTIONS \& REPLIES}

For balance, we ought to take this opportunity to note some consequences of the intuitionistic semantics presented above. Those skeptical of intuitionism will no doubt be inclined to view these consequences as failures of our semantics or of my argumentation. Although I shall not attempt a full rebuttal of these claims here, I hope to give some indication of how the intuitionist might respond.

First, it should be noted that, speaking precisely, the intuitionist should say that sentences like OPN or Goldbach's Conjecture are not currently $\mathbf{T}$; they have not been proven, but perhaps they might be proven someday. For this very reason, the intuitionist schema must be dynamic, allowing sentences to change their truth-value across time. While there is certainly something odd about the suggestion that, say, Goldbach's Conjecture is not currently true, but might be true in the future, I would suggest that this oddness is a hangover from our (pre-analytic) inclination to think classically about the mathematical domain. It may be intuitive to think of mathematical statements as being determinately true or false, but this intuitiveness is not a guarantee of theoretical adequacy. As Heyting puts it, "In fact all mathematicians and even intuitionists are convinced that in some sense mathematics bear upon eternal truths, but when trying to define precisely this sense, one gets entangled in a maze of metaphysical difficulties." ${ }^{21}$

Second, as a consequence of the above, we should not admit that the negation “ $\neg \varphi$ " of a sentence " $\varphi$ " is $\mathbf{T}$ unless $\neg \varphi$ is proven; that is, under the BHK interpretation, it is proven that $\varphi$ will never be proven. In this respect, the logic of intuitionism is weaker than classical logic. It is not intuitionistically valid to infer that “ $\neg \varphi$ " is $\mathbf{T}$ from the fact that " $\varphi$ " is not-T; that is, the Law of Excluded Middle is not intuitionistically valid. This may not sit well with those schooled in classical logic, but again, I would argue that any discomfort felt here was a consequence of our tendency to think classically and to equivocate over the truth of a state of affairs $\varphi$ and the truth of the corresponding sentence " $\varphi$ " (that is, its satisfaction of the $\mathbf{T}$-predicate). If we think of the $\mathbf{T}$-predicate as signaling something closer to adequacy (without any metaphysical presuppositions), then the loss of the Law of Excluded Middle looks less objectionable. Naturally, the formal semantics necessary to explicate this logic are more complicated than for classical logic, but they can be formulated.
Third, the arguments I have presented here go no way to establishing that intuitionism is the only adequate interpretation of mathematical language, that mental construction is the only suitable interpretation of the members of the domain $\mathbf{N}$, or that proof is the only suitable notion for grounding mathematical semantics. Nor are my arguments immune from rebuttal from the classical mathematician. Such a rebuttal would most likely have to take one of two forms. First, one could accept Wittgenstein's doctrine that "meaning is use," but contend that the classical interpretation is not in contravention of this (or, possibly, only that the intuitionist is as guilty as the classicist of contravening Wittgenstein). This would leave us at an impasse with no consensus on an admissible mathematical semantics. Second, one could directly reject Wittgenstein's claim that use determines meaning, and thereby salvage a classical interpretation, even as this interpretation contravenes our actual use of mathematical language. I do not mean to argue that such rebuttals are not possible; I hope only to have shown that the burden of proof is on the critic of intuitionism to refute the intuitionistic interpretation. 


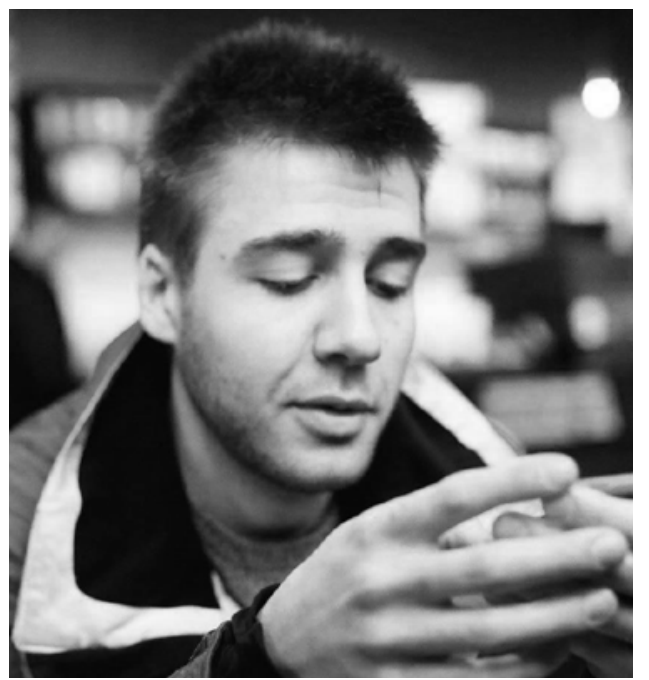

Samuel R. Elliott is a recent philosophy graduate of the University of

Edinburgh. His primary interests are

in the philosophy of language and

philosophical logic, with a secondary

interest in examining a range of

philosophical problems through the lens

of language. Outside philosophy, Sam is

an overambitious cook and vociferous

consumer of soccer. 